\title{
The effect of equations of state on the performances of compositional grading models
}

Ikechi Igwe ${ }^{1}$, Jebraeel Gholinezhad ${ }^{1}$, Mohamed Hassan ${ }^{1}$

${ }^{1}$ School of Energy and Electronic Engineering, University of Portsmouth, United Kingdom.

Corresponding author: Jebraeel Gholinezhad (jebraeel.gholinezhad@port.ac.uk), School of Energy and Electronic Engineering, Anglesea Building, University of Portsmouth, PO1 3DJ, United Kingdom.

\section{Biographical note}

Ikechi Igwe is a Ph.D student at the School of Energy and Electronic Engineering (Petroleum Engineering), University of Portsmouth, United Kingdom, and a lecturer at the Petroleum Engineering Department, Rivers state University, Port Harcourt, Nigeria. His research interest is focused on reservoir PVT analysis and reservoir modeling and simulation.

Jebraeel Gholinezhad obtained his Ph.D in Petroleum Engineering from Heriot-Watt University, Edinburgh, United Kingdom. He is a lecturer at the School of Energy and Electronic Engineering, University of Portsmouth, United Kingdom. His research interest is focused on shale gas reservoir modeling and reservoir fluid studies.

Mohamed Hassan obtained his Ph.D in Chemical Engineering from Loughborough University, United Kingdom. He is a senior Lecturer and course Leader of Petroleum Engineering, University of Portsmouth, United Kingdom. His research interest is on Petroleum fluids and shale gas processing and economics. 


\title{
The effect of equations of state on the performances of compositional grading models.
}

\author{
Abstract \\ The effect of equations of state on the performance of compositional grading \\ models to accurately predict compositional variation with depth in petroleum \\ reservoir has been presented. Compositional grading models considered are \\ isothermal model, zero thermal diffusion model, Haase's thermal diffusion \\ model, and Kempers thermal diffusion model. The performances of these models \\ was simulated based on tuned equations of state. The simulation results were \\ validated with experimental data and the associated error computed as percentage \\ average absolute deviation. The results suggest that selection of a suitable \\ equations of state has significant influence on the performances of the applied \\ compositional grading models.
}

Keywords: compositional grading; isothermal model; non-isothermal models; equations of state; simulation

\section{Introduction}

An equation of state (EOS) is a semi-empirical relationship of pressure, volume, and temperature which describe the phase behaviour of pure substances. The application of EOS to multicomponent systems such as hydrocarbon mixtures requires an extra variable - composition, and a suitable mixing rule (Danesh 1998). Mixing rules enable the description of the prevailing forces between molecules of different substances in the mixture (Danesh 1998). Despite the fact that equilibrium conditions can be scrupulously determined thermodynamically, the accuracy of phase equilibrium prediction depends mainly on the capability of the EOS and the concomitant mixing rule (Danesh 1998).

Most of the phase equilibrium calculations implemented for hydrocarbon mixtures were based on cubic EOS which dates back to the renowned work of van der Waals (vdW) in 1873 (Pederson and Christensen 2007). Redlich and Kwong (RK) EOS was the first cubic EOS to gain extensive application in 1949 (Redlich and Kwong 1949; 
Pederson and Christensen 2007). They introduced a temperature dependency to the attraction parameter " $\alpha$ " of the vdW EOS. Several other modifications of the vdW EOS have been reported in the literature (Thiele 1963; Carnahan and Starling 1972; Chien, Greenkorn and Chao 1983; Adachi and Lu 1984; Watson et al. 1986; Lin et al. 1985; Wei and Sadus 2000; Span et al. 2001; Tian and Gui 2003). The RK EOS was further modified by Soave, and Peng and Robinson (Soave 1972; Peng and Robinson 1976; Robinson and Peng 1978). Soave modified the RK EOS by replacing the temperature dependency of the attractive term in RK EOS with Pitzer's acentric factor to develop the original Soave-Redlich-Kwong (SRK) equation of state (Zudkevitch, and Joffe, 1970; Joffe, et al., 1970; Soave 1972). The acentric factor was introduced to enable prediction of hydrocarbon vapour pressure. Grabowski and Daubert also modified the original SRK EOS by formulating a new expression for the constant " $\alpha$ " (Grabowski and Daubert 1978). This new modification is known as SRK (G\&D) EOS. Peng and Robinson (PR) modified the RK EOS by proposing an improved expression for the attractive term primarily to improve liquid density prediction (Zudkevitch, and Joffe, 1970; Joffe, et al., 1970; McCain, 1990). PR EOS shows performance analogous to SRK EOS, although, it is usually superior in calculating liquid densities of particularly nonpolar substances (Danesh 1998). Several other modifications mainly aimed at improving performances of the various cubic EOSs have been reported in the literature (Harmens and Knapp 1980; Schmidt and Wenzel 1980; Patel and Teja 1982; Péneloux, Rauzy and Fréze 1982; Mathias 1983; Yu and Lu 1987; Valderrama 1990; Twu et al. 1991; Tsai and Chen 1998; Ahlers and Gmehling 2001; Cismondi and Mollerup 2005; Haghtalabet al. 2010; Abudour et al. 2012; Burgess et al. 2013; Mahmoodi 2016; Privat et al. 2016). 
While an EOS mainly generates volumetric data, its main application in engineering is through its coupling with thermodynamic models in estimating phase behaviour and physical properties of fluids (Danesh 1998). Compositional grading (CG) models are typical examples of such thermodynamic relations which are usually coupled with EOS to predict hydrocarbon compositional variation and physical properties variation with depth in oil and gas reservoirs. These CG models are classified as either isothermal model or non-isothermal models. The effect of gravity on hydrocarbon component gradation is modeled based on isothermal CG model (Sage and Lacey 1939; Schulte 1980; Whitson and Belery 1994; Boukadi, Bemani and Rumhy 1999; Høier and Whitson 2000; Pedersen and Hjermstad 2006) while the effects of thermal diffusion and temperature gradient are modeled based on various non-isothermal CG models (Dougherty and Drickamer 1955; Belery and da Silva 1990; Chaback 1992; Whitson and Belery 1994; Høier and Whitson 2000; Nikpoor, Kharrat and Chen 2011: Pedersen and Hjermstad 2015). Comparison of the performances of isothermal model and nonisothermal models - zero thermal diffusion model; Haase thermal diffusion model; and Kemper's thermal diffusion model, based on a single EOS, have been presented in the literature (Høier and Whitson 2000). Uninformed Selection of a suitable EOS for appropriate reservoir fluid characterization can lead to significant errors in estimated fluid compositional gradient, reservoir pressure gradient, and saturation pressure gradient, which would, in turn, result in poor economic and field development decisions.

Therefore, the aim of this work is to simulate the influence of different EOSs on the performances of CG models to accurately predict compositional variation with depth in petroleum reservoir. This will ensure that suitable EOS is selected for a particular CG model. CG models evaluated include isothermal model, zero (passive) thermal diffusion 
model, Haase's thermal diffusion model, and Kempers thermal diffusion model. Other non-isothermal CG models such as Doughtery-Drickamer (Berlary-da silva modification) model and Firoozabadi-Ghorayeb model were not considered in this study because they are not supported by the applied CMG WinProp simulator. To include these two models in this study would require developing separate solution algorithms for each of them and the related EOSs which is beyond the scope of this study.

More so, in an exceptional case where all the above mentioned CG models have been used for analysis by Høier and Whitson (2000), the authors were not directly responsible for the results obtained by the application of Firoozabadi-Ghorayeb model. Høier and Whitson (2000) reported that the result presented from application of Firoozabadi-Ghorayeb model was provided by a third party. This suggest that no single research effort has been able to apply all the various CG models. The list of available non-isothermal CG models is rather extensive. For example, Esposito et al. (2017) presented CG calculations based on non-isothermal models other than the above mentioned models. The non-isothermal models applied by Espsito et al. (2017) include Pederson and Linderloff (2003) model, Pedersen and Hjermstad (2006) model, and Ghorayeb et al. (2003) model. Hence, the scope of this current study is limited to isothermal model, passive thermal diffusion model and static thermal diffusion models (Haase's model and Kempers model).

\section{Theory}

The mathematical framework for the estimation of compositional variation with depth in petroleum reservoirs under the influence of chemical potential, gravitational force, and thermal diffusion were presented by Montel and Gouel (1985); Montel (1993); Faissat et al. (1994); Høier and Whitson (2000). The one-dimensional zero-mass-flow 
stationary state model proposed by Høier and Whitson (2000) can be written as shown in Eq. (1) for the combined effects of gravity and thermal diffusion:

$$
\nabla \mu_{i}=J_{G i}-J_{T i} \nabla T / T
$$

In Eq. (1), $\mu_{i}$ is the chemical potential of component $i, \nabla T$ is the temperature gradient, $T$ is the system temperature, $h$ is the depth of interest, $J_{G i}$ accounts for the effect of gravity, and $J_{T i}$ denotes thermal diffusion factor. The term $J_{G i}$ is expressed thus:

$$
J_{G i}=\left(M_{i}-\rho V_{i}\right) g
$$

where $M_{i}$ is the molecular weight of component $i, V_{i}$ is the partial molar volume of component $i, \rho$ is mass density, and $g$ is acceleration due to gravity.

One necessary constraint to the application of Eq. (1) either in the form of isothermal model or non-isothermal models is that the sum of the molar compositions of all fluid components at a given depth along the hydrocarbon column must add up to one (unity). That is:

$$
\sum_{i=1}^{n} z_{i}(h)=1
$$

where $n$ is the number of components and $z_{i}$ is the overall composition of component $i$. Hence, there are $n+1$ variables at any given depth along the hydrocarbon column. To determine the pressure and molar compositions at any depth will consequently require solving $n+1$ equations consisting $n+1$ variables, with suitable EOS. Eq. (1) is the compositional grading model used in this work. 


\section{Isothermal CG model}

Isothermal CG model assumes thermodynamic equilibrium conditions in the reservoir. It neglects the effect of thermal gradient and thermal diffusion $\left(J_{T i}=0\right)$. Thus, gravity force is the only factor responsible for the distribution of fluid compositions in the reservoir, causing lighter components like methane to migrate towards the top of the reservoir and heavier components to move towards the bottom section. Eq. (1) can be mathematically transformed to an isothermal model by expressing it in terms of component fugacity as follows:

$$
f_{i}(h)=f_{i}\left(h^{o}\right) \exp \left(-\frac{M_{i} g\left(h-h^{o}\right.}{R T}\right) \quad i=1,2, \ldots, n
$$

The fugacity of component $i$ can be estimated based on the overall composition of the species, thus:

$$
f_{i}=p_{i} z_{i} \varphi_{i}
$$

Therefore, Eq. (4) can be rewritten thus:

$$
\left(\varphi_{i}^{h} z_{i}^{h} p_{i}^{h}\right)=\left(\varphi_{i}^{h^{o}} z_{i}^{h^{o}} p_{i}^{h^{o}}\right) \exp \left(-\frac{M_{i} g\left(h-h^{o}\right.}{R T}\right) \quad i=1,2, \ldots, n
$$

In Eq. (4) - (6), $f_{i}$ is the fugacity of component $i, h^{o}$ is the reference depth, $h$ is the depth of interest, $R$ is the acceleration due to gravity, $p_{i}$ is the pressure of component $i$, $\varphi_{i}$ is the fugacity coefficient of component $i$, and all other terms are as defined in Eq. (1). Eq. (6) is the isothermal CG model applied in this work to simulate compositional variation with depth. 


\section{Non-isothermal models}

Thermodynamic equilibrium conditions are seldom the case in most petroleum reservoirs. Petroleum reservoirs with a characteristic temperature gradient are referred to as non-isothermal reservoirs and will not be at thermodynamic equilibrium. The presence of temperature gradient in the reservoir will lead to the migration of heavier components to the top of the reservoir and lighter fluid components to the hot bottom zone. Therefore, thermal diffusion opposes the effect of gravity.

Non-isothermal models considered in this work are the zero thermal diffusion model, Haase's thermal diffusion model, and Kempers thermal diffusion model. Zero (passive) thermal diffusion model is a hypothetical model in which the thermal diffusion factor $\left(J_{T i}\right)$ in Eq. (1) is assumed to be negligible even though thermal gradient exist in the system $\left(J_{T i}=0, \nabla T \neq 0\right)$. The temperature, $T$, at a depth $(h)$ is estimated from the knowledge of temperature gradient $(\nabla T)$. By assuming $J_{T i}=0$ but accounting for thermal gradient, Eq. (1) is solved by integrating with depth discretization using applied computer simulator.

The basic difference between the various thermal diffusion models is in the method applied in estimating the thermal diffusion coefficient $\left(J_{T i}\right)$ in Eq. (1). Haase's thermal diffusion model relied on the centre of mass assumption to estimate the thermal diffusion coefficient as follows:

$$
J_{T i}=1 / M_{i} \cdot\left(M_{i} H_{m}-M_{m} H_{i}\right)
$$

Where

$$
\begin{aligned}
& M_{m}=\sum_{i} x_{i} M_{i} \\
& H_{m}=\sum_{i} x_{i} H_{i}
\end{aligned}
$$


$M_{m}$ is the molecular weight of the mixture, $H_{m}$ is the molar enthalpy of the mixture, $M_{i}$ is the molecular weight of component $i$ in the mixture, $H_{i}$ is the partial molar enthalpy of component $i$ in the mixture, $x_{i}$ is the mole fraction of component $i$.

Kempers thermal diffusion model depends on the centre of volume assumption for thermal diffusion coefficient prediction. It is expressed thus:

$$
J_{T i}=1 / V_{i} \cdot\left(V_{i} H_{m}-V_{m} H_{i}\right)
$$

where

$$
V_{m}=\sum_{i} x_{i} V_{i}
$$

In Eq. (10) and (11), $V_{m}$ is the molar volume of the mixture, $V_{i}$ is the partial molar volume of component $i$ in the mixture.

\section{Experimental data}

Experimental data comprising gas condensate reservoir fluid composition and related reservoir properties at a reference depth as reported by Pedersen and Hjermstad (2015) have been analysed. The reservoir fluid composition and properties at a reference (sample) depth of $175 \mathrm{~m}$ are presented in Table 1 while Table 2 shows the measured (experimental) $\mathrm{C}_{1}$ and $\mathrm{C}_{10+}$ mole $\%$ at different depths in the study reservoir. 
Table 1. Reservoir fluid composition at a reference depth of $175 \mathrm{~m}$ (Pedersen and Hjermstad 2015)

\begin{tabular}{lc}
\hline Component & Mole \% \\
\hline $\mathrm{N}_{2}$ & 0.42 \\
$\mathrm{CO}_{2}$ & 0.69 \\
$\mathrm{C}_{1}$ & 50.04 \\
$\mathrm{C}_{2}$ & 7.85 \\
$\mathrm{C}_{3}$ & 6.77 \\
$\mathrm{iC}_{4}$ & 1.04 \\
$\mathrm{nC}_{4}$ & 3.2 \\
$\mathrm{iC}_{5}$ & 1.16 \\
$\mathrm{nC}_{5}$ & 1.55 \\
$\mathrm{C}_{6}$ & 1.88 \\
$\mathrm{C}_{7}$ & 3.5 \\
$\mathrm{C}_{8}$ & 3.75 \\
$\mathrm{C}_{9}$ & 2.28 \\
$\mathrm{C}_{10+}$ & 15.88 \\
\hline $\mathrm{C}_{10+}$ molecular weight $(\mathrm{MW})$ & 196 \\
$\mathrm{C}_{10+}$ specific gravity & 0.85 \\
$\mathrm{Reservoir}$ & 28400 \\
$\mathrm{Reserv}$ & 93 \\
Saturation pressure $(\mathrm{kPa})$ & 27200 \\
Depth to top of reservoir $(\mathrm{kPa})$ & 0 \\
Depth to bottom of reservoir $(\mathrm{m})$ & 327 \\
\hline
\end{tabular}

Table 2. Measured (experimental) $\mathrm{C}_{1}$ and $\mathrm{C}_{10+}$ mole $\%$ at different depths in the reservoir (Pedersen and Hjermstad 2015)

\begin{tabular}{lccccc}
\hline & \multicolumn{5}{c}{ Depth (m) } \\
\cline { 2 - 6 } Components & 0 & 175 & 204 & 228 & 327 \\
mole \%o & \\
\hline $\mathrm{C}_{1}$ & 75.66 & 50.04 & 49.88 & 48.89 & 45.66 \\
$\mathrm{C}_{10+}$ & 1.57 & 15.88 & 16.11 & 16.70 & 17.66 \\
\hline
\end{tabular}

\section{Simulation procedure}

Modeling the influence of EOSs on the performances of isothermal and non-isothermal CG models is performed in WinProp 2017 version. WinProp is the Computer Modeling Group's (CMG's) EOS multiphase equilibrium property simulator. The modeling process starts with the selection of EOS, specification of unit and feed (mole or mass). The four EOSs considered are the PR 1976 EOS with 1976 expression for the constant “ $\alpha ”$, PR 1978 EOS with 1978 expression for the constant “ $\alpha$ ”, the original SRK EOS, and SRK EOS with the constant " $\alpha$ " proposed by Grabowski and Daubert (SRK 
(G\&D)). These EOSs were selected for the following reasons: they are the only EOSs available in the applied commercial simulator - CMG WinProp; and they are the most commonly used EOSs in the petroleum industry due to their applicability to multicomponent systems (Firoozabadi 1988; Esmaeilzadeh et al. 2005; Ashouri et al. 2011). The next step in the simulation process involves selection of the reservoir fluid components and estimation of their respective physical and critical properties. The decane plus fraction $\left(\mathrm{C}_{10^{+}}\right)$was defined using its molecular weight and specific gravity.

Thereafter, the composition of each fluid component either in mole fraction or percentage, are specified. During this step, the model constraint stated in Eq. (3), is complied with by ensuring that the sum of the mole fraction of all fluid components equals unity or $100 \%$. Regression (tuning) of the EOSs parameters is implemented subsequently. The objective of regression is to minimize the square error between EOS predicted results and the experimental values. WinProp uses the Agarwal et al. (1990) regression procedure to tune the EOSs. This procedure ensures that the most sensitive parameter amongst selected parameters is regressed first. Regression parameters used include the critical pressure and temperature of $\mathrm{C}_{10+}$, acentric factor of $\mathrm{C}_{10+}$, molecular weight of $\mathrm{C}_{10+}$, hydrocarbon interaction coefficient exponent, and the interaction coefficient between $\mathrm{C}_{10+}$ and $\mathrm{CO}_{2}$. The simulator default convergence tolerance of 1E06 and 99 maximum number of iterations were adopted.

Parameters required for the calculation of saturation pressure, liquid densities, and phase stability were then specified. These parameters include the temperature at which the saturation pressure is to be calculated, initial saturation pressure estimate, and experimental value of the saturation pressure that can be matched by tuning. The final step in the modeling process is the coupling of selected CG models (isothermal model, zero thermal diffusion model, Haase's thermal diffusion model, and Kempers thermal 
diffusion model) with the EOS model and specification of values for the various parameters in the CG models presented in Eq. (1) - (11). A vertical temperature gradient of $0.025{ }^{\circ} \mathrm{C} / \mathrm{m}$ which was proposed by Pedersen and Hjermstad (2015) was applied to the non-isothermal models. At this juncture, the coupled models are executed separately and the simulation results recorded.

Statistical analysis of the results based on percentage average absolute deviation (\% AAD) was performed to determine the error between simulation results and the experimental (measured) values. The lower the calculated \% AAD, the less the calculated error, hence, the more suitable the related EOS is to the applied CG model. The mathematical expression of the \% AAD is presented in Eq. (12):

$$
\% A A D=100 * A B S\left[\frac{\sum_{i=1}^{N}\left(\left(n_{i(\bmod e l)}-n_{i(\exp )}\right) / n_{i(\exp )}\right)}{N}\right]
$$

Where $n_{i(\bmod e l)}$ is the model predicted mole fraction of component $i$ with respect to depth, $\boldsymbol{n}_{i(\exp )}$ is the experimental (measured) mole fraction of component $i$ with respect to depth, and $N$ is the number of sample points (observations).

\section{Results and Discussion}

Although, the applied reservoir fluid composition consists of fourteen (14) fluid components, only $\mathrm{C}_{1}$ and $\mathrm{C}_{10+}$ were selected as the key components to investigate the influence of the various EOSs on the performances of the applied CG models. This is because $\mathrm{C}_{1}$ and $\mathrm{C}_{10+}$ represents the most dominant lighter and heavier components, respectively. 
The effect of EOSs on the performance of isothermal CG model to accurately predict $\mathrm{C}_{1}$ and $\mathrm{C}_{10+}$ variation with depth are presented in Figures 1 and 2, respectively. Figure 1 shows that there are marginal differences in the trends indicated by the various EOSs within the top zone of the reservoir. The various EOS models exhibited similarity in $\mathrm{C}_{1}$ trends towards the bottom section of the reservoir with no significant difference. The calculated $\%$ AAD (statistical analysis) between the experimental values of $\mathrm{C}_{1}$ mole $\%$ at different depth and the values predicted by the different EOSs based CG models are presented in Table 3 .

The calculated $\%$ AAD with respect to Figure 2 is presented in Table 3 . Table 3 shows that PR 1976 EOS generated the least \% AAD for both $\mathrm{C}_{1}$ and $\mathrm{C}_{10+}$ gradations. These results suggest that in order to accurately predict CG using isothermal CG model, the most realistic EOS model for fluid characterization is PR 1976.

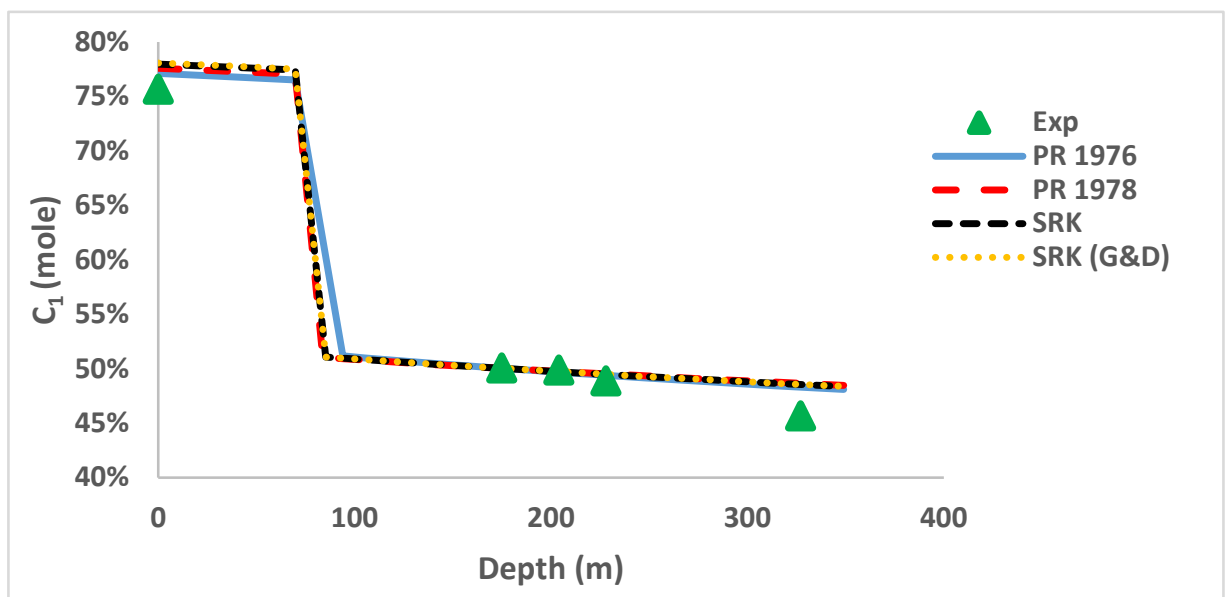

Figure 1. Comparison of the effect of different EOSs on the performance of isothermal CG model to accurately predict $\mathrm{C}_{1}$ variation with depth 


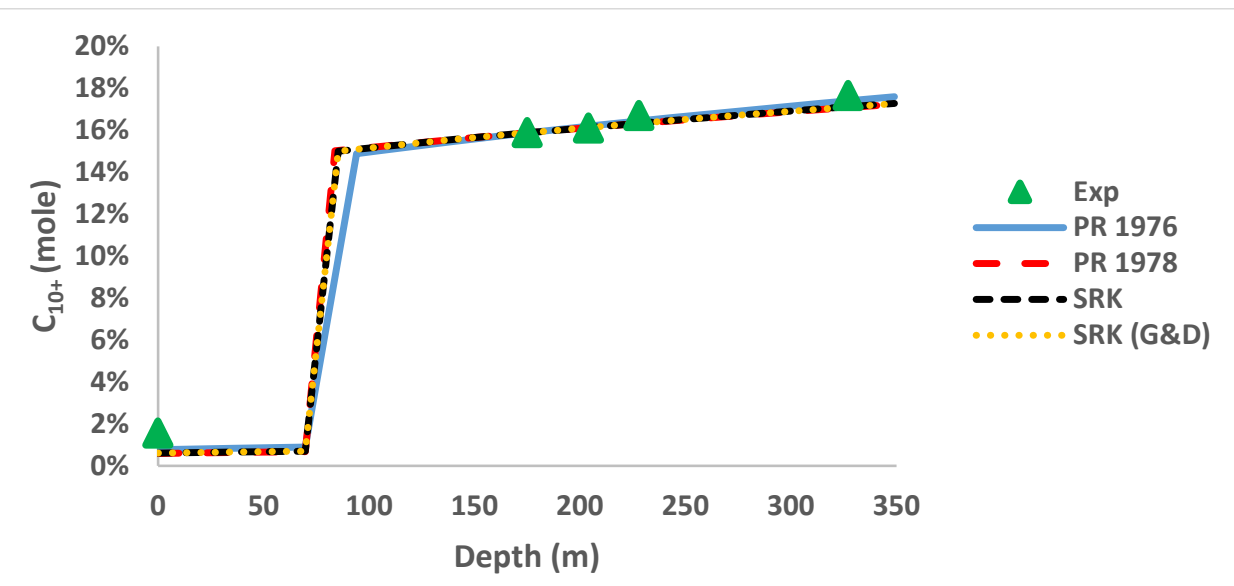

Figure 2. Comparison of the effect of different EOSs on the performance of isothermal $\mathrm{CG}$ model to accurately predict $\mathrm{C}_{10+}$ variation with depth

Table 3. Comparison of \% AAD predicted by various EOSs based isothermal CG model

\begin{tabular}{lcc}
\hline EOS & \% AAD for $\mathbf{C}_{\mathbf{1}}$ & \% AAD for $\mathbf{C}_{\mathbf{1 0 +}}$ \\
\hline PR 1976 & 1.84 & 10.75 \\
PR 1978 & 1.90 & 11.15 \\
SRK & 2.19 & 13.27 \\
SRK (G\&D) & 2.22 & 13.20 \\
\hline
\end{tabular}

Figures 3 and 4 presents the effect of EOSs on the performance of zero thermal diffusion $\mathrm{CG}$ model to accurately predict $\mathrm{C}_{1}$ and $\mathrm{C}_{10+}$ variation with depth, respectively. PR 1976 and PR 1978 predicted the most accurate $C_{1}$ gradation at the reservoir top while at the bottom, all the EOS models overestimated $\mathrm{C}_{1}$ gradation as can be seen in Figure 3. Figure 4 shows that at both the top and bottom sections of the reservoir, the $\mathrm{C}_{10+}$ gradation predicted by the various EOS models were all in significant agreement with the measured trend. However, statistical analysis of Figures 3 and 4 presented in Table 4 confirmed that each EOS predicted results generated different values of \%AAD with PR 1976 EOS estimating the least \% AAD. Hence, PR 1976 is also the most suitable EOS for zero thermal diffusion CG model. 


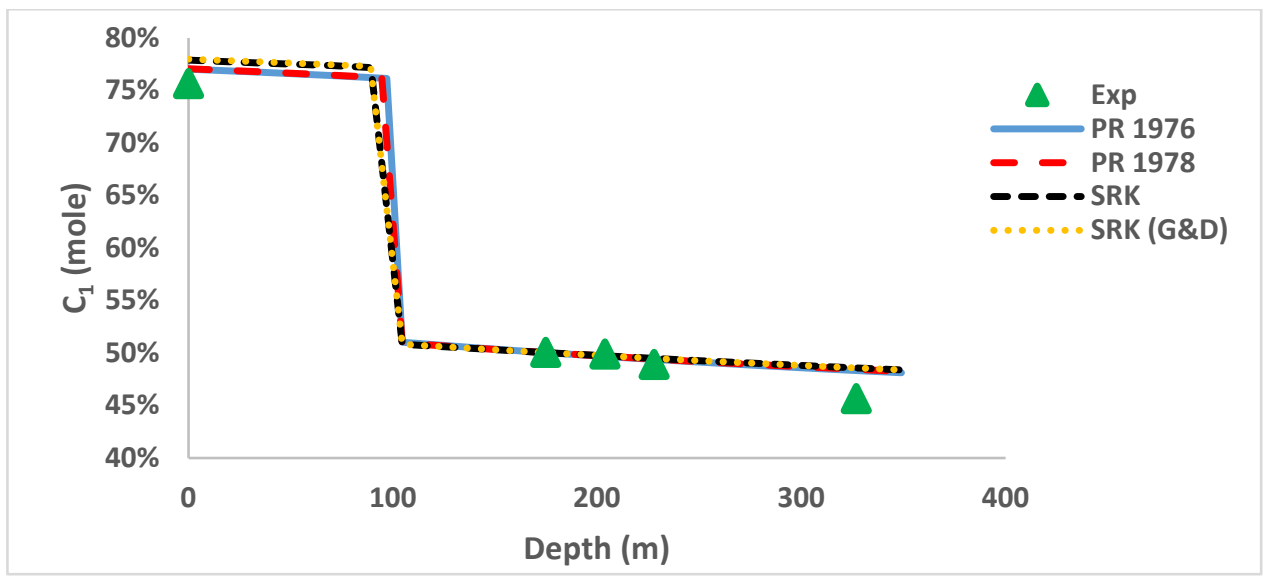

Figure 3. Comparison of the effect of different EOSs on the performance of zero thermal diffusion $\mathrm{CG}$ model to accurately predict $\mathrm{C}_{1}$ variation with depth

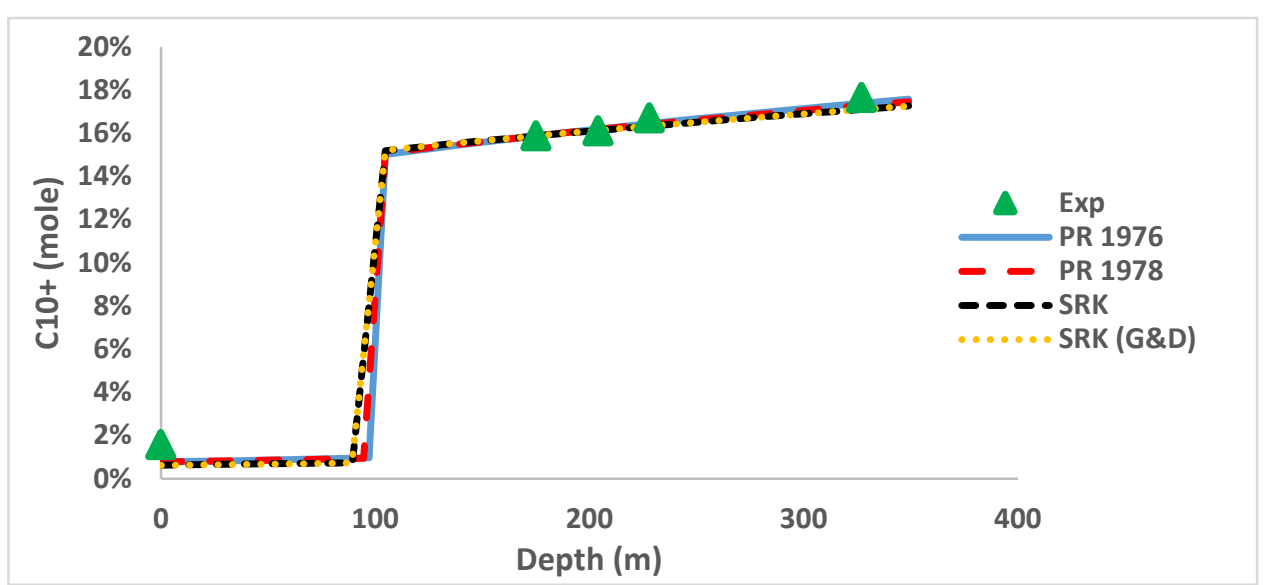

Figure 4. Comparison of the effect of different EOSs on the performance of zero thermal diffusion $\mathrm{CG}$ model to accurately predict $\mathrm{C}_{10+}$ variation with depth

Table 4. Comparison of \% AAD predicted by various EOSs based zero thermal diffusion CG model

\begin{tabular}{lcc}
\hline EOS & \% AAD for $\mathbf{C}_{\mathbf{1}}$ & \% AAD for $\mathbf{C}_{\mathbf{1 0 +}}$ \\
\hline PR 1976 & 1.82 & 10.62 \\
PR 1978 & 1.89 & 10.92 \\
SRK & 2.17 & 15.54 \\
SRK (G\&D) & 2.20 & 13.22 \\
\hline
\end{tabular}

The performances of various EOSs based Haase's thermal diffusion CG model to accurately predict $\mathrm{C}_{1}$ and $\mathrm{C}_{10}$ variation with depth are presented in Figures 5 and 6 , respectively. It is evident from Figure 5 that at the top of the reservoir, SRK and SRK (G\&D) predicted similar $\mathrm{C}_{\mathrm{I}}$ gradation trends that is somewhat less accurate than the trends exhibited by PR 1976 and PR 1978. Towards the bottom section of the reservoir, all the EOS models predicted $\mathrm{C}_{1}$ gradation with significantly similar degree of accuracy. 
The simulated gradation of $\mathrm{C}_{10+}$ illustrated in Figure 6 suggest similarity in the trends predicted by the various EOS models at both the top and bottom sections of the reservoir. Despite the observed similarities, statistical analysis of Figures 5 and 6 presented in Table 5 inveterate significant difference in the values of calculated $\%$ AAD and shows that PR 1987 is the most suited EOS for Haase's thermal diffusion CG model.

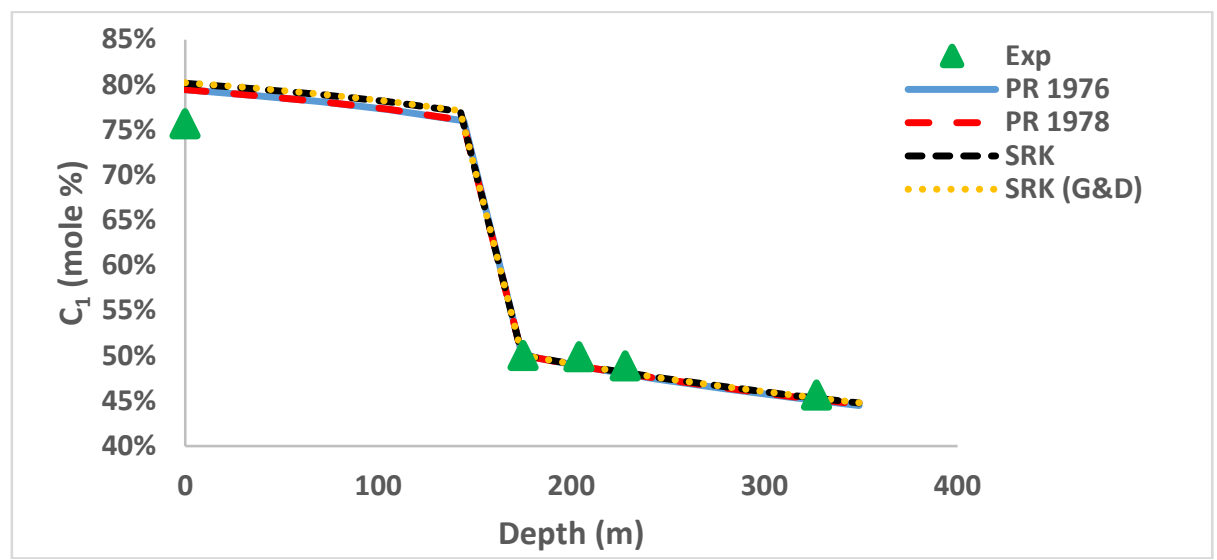

Figure 5. Comparison of the effect of different EOSs on the performance of Haase's thermal diffusion $\mathrm{CG}$ model to accurately predict $\mathrm{C}_{1}$ variation with depth

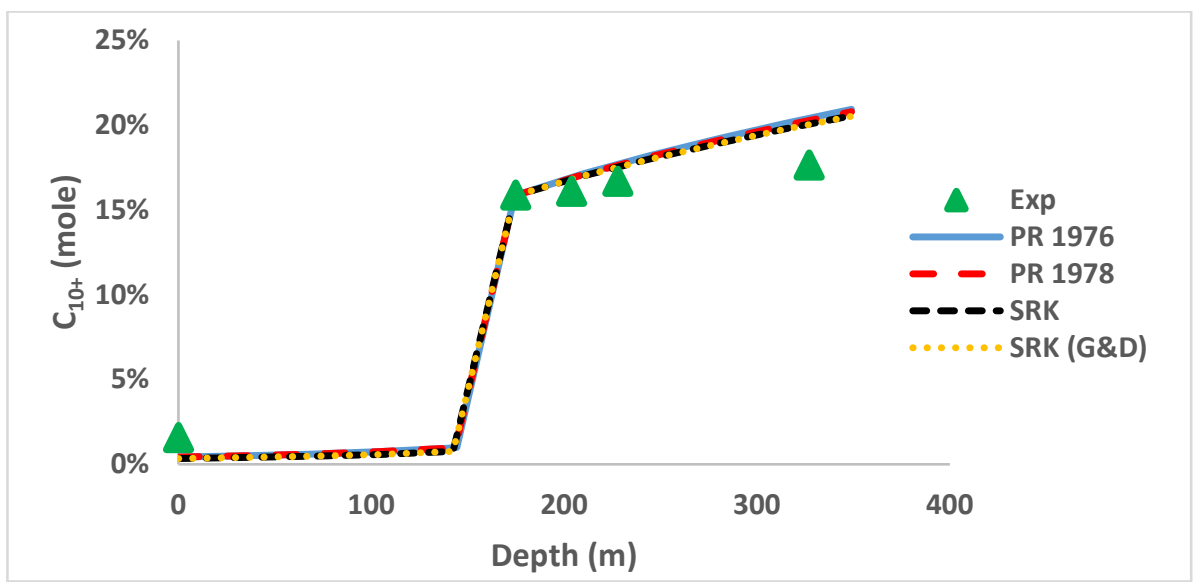

Figure 6. Comparison of the effect of different EOSs on the performance of Haase's thermal diffusion $\mathrm{CG}$ model to accurately predict $\mathrm{C}_{10+}$ variation with depth 
Table 5. Comparison of \% AAD predicted by various EOSs based Haase's thermal diffusion CG model

\begin{tabular}{lcc}
\hline EOS & \% AAD for $\mathbf{C}_{\mathbf{1}}$ & \% AAD for $\mathbf{C}_{\mathbf{1 0 +}}$ \\
\hline PR 1976 & 2.08 & 19.89 \\
PR 1978 & 1.97 & 19.61 \\
SRK & 2.04 & 20.31 \\
SRK (G\&D) & 2.04 & 20.22 \\
\hline
\end{tabular}

The simulated results of the effect of the applied EOSs on the performance of Kempers thermal diffusion CG model are presented in Figures 7 and 8. The $C_{1}$ and $C_{10+}$ gradations are compared with the measured values as shown in Figures 7 and 8, respectively. Figures 7 and 8 shows that the $\mathrm{C}_{1}$ and $\mathrm{C}_{10+}$ gradation trends predicted by the various EOS models exhibited analogous trends to the trends presented in Figures 5 and 6 (Haase's thermal diffusion CG model) but with distinct \% AAD values. The computed $\%$ AAD of the various EOSs are presented in Table 6 . Table 6 shows that PR 1976 EOS with the least \% AAD, is the most suitable EOS for Kemper's thermal diffusion CG model.

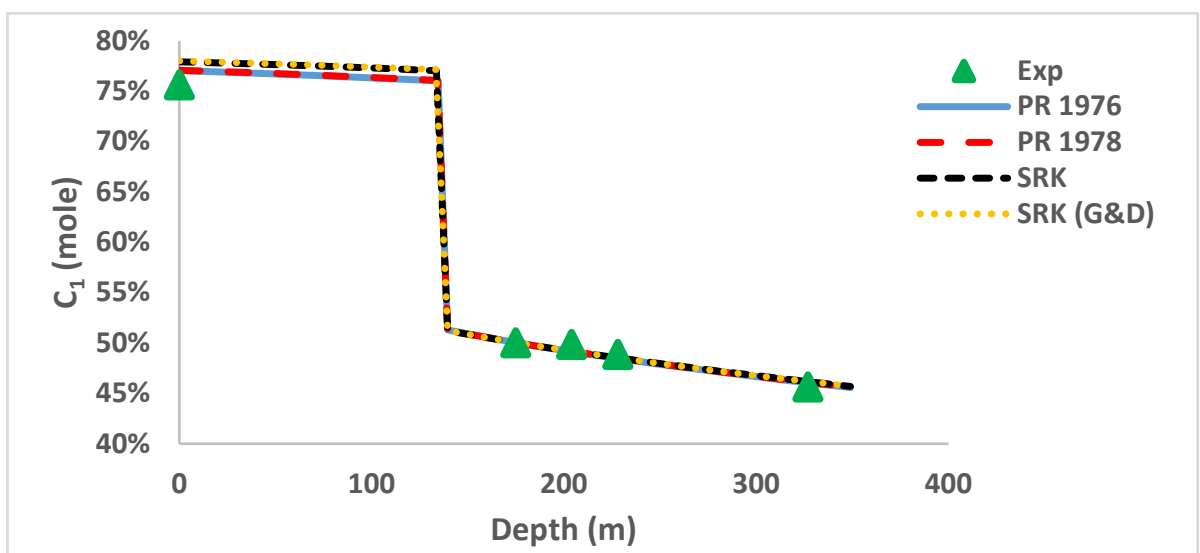

Figure 7. Comparison of the effect of different EOSs on the performance of Kempers thermal diffusion $\mathrm{CG}$ model to accurately predict $\mathrm{C}_{1}$ variation 


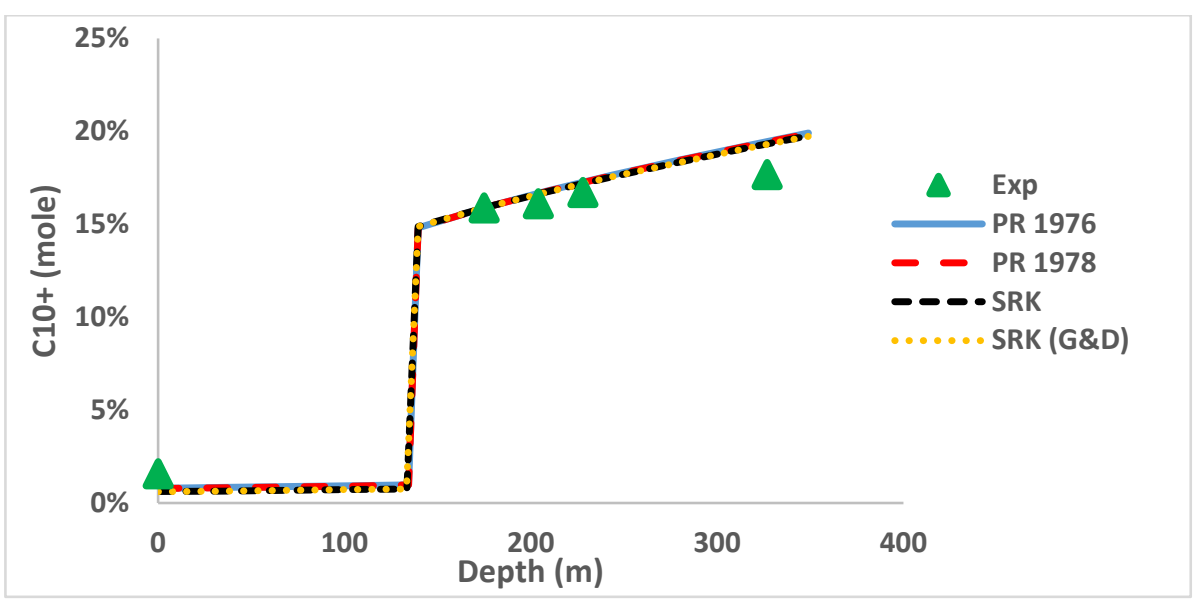

Figure 8. Comparison of the effect of different EOSs on the performance of Kempers thermal diffusion $\mathrm{CG}$ model to accurately predict $\mathrm{C}_{10+}$ variation with depth

Table 6. Comparison of \% AAD predicted by various EOSs based Kempers thermal diffusion CG model

\begin{tabular}{lcc}
\hline EOS & \% AAD for $\mathbf{C}_{\mathbf{1}}$ & \% AAD for $\mathbf{C}_{\mathbf{1 0 +}}$ \\
\hline PR 1976 & 1.01 & 13.34 \\
PR 1978 & 1.04 & 13.40 \\
SRK & 1.26 & 15.23 \\
SRK (G\&D) & 1.28 & 15.16 \\
\hline
\end{tabular}

\section{Conclusion}

Simulation results from the applied CG models (isothermal model, zero thermal diffusion model, Haase's thermal diffusion model, and Kempers thermal diffusion model) and statistical analysis of the results (\%AAD) corroborated the fact that selection of a suitable EOS has significant effect on the performances of the applied CG models. Coupling PR 1976 EOS with isothermal model, zero thermal diffusion model, and Kempers thermal diffusion model, respectively, resulted to superior performance of the models. Amongst the applied EOSs for Haase's thermal diffusion model, PR 1978 EOS simulated the most accurate compositional grading with respect to experimental values.

\section{Acknowledgement}

We honestly acknowledge the anonymous reviewers whose corrections and comments 
have significantly enhanced the quality of this manuscript. This work was supported by Tertiary Education Trust Fund (TETFund) of Nigeria, under the academic staff training and development scholarship award (2014/2015).

\section{Disclosure statement}

There is no conflict of interest to declare.

\section{SI metric conversion factors}

${ }^{\circ} \mathrm{C}+273.15 \quad=\mathrm{K}$

\section{Reference}

Abudour, A. M., S. A. Mohammad, R. L. Robinson Jr., and K. A .M. Gasem. 2012. Volume translated Peng-Robinson equation of state for saturated and single-phase liquid densities. Fluid Phase Equilibria 335: 74-87. doi:10.1016/j.fluid.2012.08. 013.

Adachi, Y., and B. C.-Y. Lu. 1984. Simplest equation of state for vapor-liquid equilibrium calculation: a modification of the van der Waals equation. AIChE Journal 30(6):991-993. doi:10.1002/aic.690300619.

Agarwal, R. K., Y.-K. Li, and L. Nghiem. 1990. A regression technique with dynamic parameter selection for phase-behavior matching. SPE Reservoir Engineering 5(1):115-120. doi:10.2118/16343-PA.

Ahlers, J., and J. Gmehling. 2001. Development of an universal group contribution equation of state I. Prediction of liquid densities for pure compounds with a volume translated Peng-Robinson equation of state. Fluid Phase Equilibria 191: 177-188. doi:10.1016/S0378-3812(01)00626-4.

Ashour, I., N. Al-Rawahi, A. Fatemi, and G. Vakili-Nezhaad. 2011. Applications of equations of state in the oil and gas industry, thermodynamics - kinetics of dynamic systems, Dr. Juan Carlos Moreno Piraj $\tilde{A}_{i}$ (ed.), ISBN: 978-953-307-627-0. Rijeka, Croatia: InTech, available from:

http://www.intechopen.com/books/thermodynamics-kinetics-of-dynamicsystems/applications-of-equations-ofstate-in-the-oil-and-gas-industry

Belery, P., and F. V. da Silva. 1990. Gravity and thermal diffusion in hydrocarbon reservoirs. Paper presented at the Third Chalk Research Program, Copenhagen, June 11-12.

Boukadi, F., A. Bemani, and M. Rumhy. 1999. Effect of PVT properties variation with depth in hydrocarbon reservoirs, Petroleum Science and Technology 17(1-2):81-98, doi:10.1080/10916469908949708.

Burgess W. A., D. Tapriyal, B. D. Morreale, Y. Soong, H. O. Baled, R. M. Enick, Y. Wu, B. A. Bamgbade, and M. A. McHugh. 2013. Volume-translated cubic EoS and PC-SAFT density models and a free volume-based viscosity model for hydrocarbons at extreme temperature and pressure conditions. Fluid Phase Equilibria 329: 38-44. doi:10.1016/j.fluid.2013.07.016. 
Chaback, J. J. 1992. Discussion of treatment of variations of composition with depth in gas-condensate reservoirs. SPE reservoir engineering 7(1): 157-158.

Chien, C. H., R. H. Greekorn, and K. C. Chao. 1983. A chain of rotators equation of state. AIChE Journal 29(4):560-571.

Carnahan, N. F., and K. E. Starling. 1972. Intermolecular repulsions and the equation of state for fluid. AIChE Journal 18(6):1184-1189.

Cismondi, M., and J. Mollerup. 2005. Development and application of a threeparameter RK-PR equation of state. Fluid Phase Equilibria 232(1-2):74-89. doi:10.1016/j.fluid.2005.03.020

Danesh, A. 1998. PVT and phase behaviour of petroleum reservoir fluids, Vol. 47. London: Elsevier.

Dougherty, E. L., and H. G. Drickamer. 1955. Thermal diffusion and molecular motion in liquids. The Journal of Physical Chemistry 59(5):443-449. doi:10.1021/j150527a016.

Esmaeilzadeh, F., and M. Roshanfekr. 2006. A new cubic equation of state for reservoir fluids. Fluid Phase Equilibria, 239(1):83-90.

Esposito, R. O., P. H. R. Alijó, J. A. Scilipoti, and F. W. Tavares. 2017. Compositional Grading in Oil and Gas Reservoirs. Cambridge (MA): Gulf Professional Publishing.

Faissat, B., K. Knudsen, E. H. Stenby, and F. Montel. (1994). Fundamental statements about thermal diffusion for a multicomponent mixture in a porous medium. Fluid Phase Equilibria, 100:209-222.

Firoozabadi, A. 1988. Reservoir-Fluid phase behavior and volumetric prediction with equations of state (includes associated papers 18400 and 18579). Journal of petroleum technology, 40(04):397-406.

Ghorayeb K, Firoozabadi A, Anraku T. 2003. Interpretation of the unusual fluid distribution in the Yufutsu gas-condensate field. SPE Journal 8(2):114-123.

Grabowski, M. S., and T. E. Daubert.1978. A modified soave equation of state for phase equilibrium calculations. 1. Hydrocarbons systems. Industrial and Engineering Chemistry Process Design Development 17(4):448-453.

Harmens, A., and H. Knapp. 1980. Three-parameter cubic equation of state for normal substances. Industrial and Engineering Chemistry Fundamentals 19(3):291-294. doi:10.1021/i160075a010.

Haghtalab, A., M. J. Kamali, S. H. Mazloumi, and P. Mahmoodi. 2010. A new three parameter cubic equation of state for calculation physical properties and vapor-liquid equilibria. Fluid Phase Equilibria 293(2):209-218. doi:10.1016/j.fluid.2010.03.029.

Høier, L., and C. H. Whitson. 2000. Compositional grading - theory and practice. Paper presented at: Society of Petroleum Engineers Annual Technical Conference and Exhibition; October 1-4; Dallas, Texas. doi:10.2118/63085-MS.

Joffe, J., G. M. Schroeder, and D. Zudkevitch. (1970). Vapor-liquid equilibria with the redlich-kwong equation of state. AIChE Journal, 16(3):496-498.

Lin, H. M., H. Kim, T. M. Guo, and K. C. Chao. 1985. Cubic chain of rotators equation of state and VLE calculations. Fluid Phase Equilibria 13: 143-152.

McCAIN, W. D. (1990). The properties of petroleum fluids. Tulsa. Oklahoma, PennWell.

Mahmoodi, P. 2016. Soave alpha function at supercritical temperatures. The Journal of Supercritical Fluids 112: 22-36. doi:10.1016/j.supflu.2016.01.004.

Mathias, P. M. 1983. A versatile phase equilibrium equation of state. Industrial and Engineering Chemistry Process Design and Development 22(3):385-391. doi:10.1021/i200022a008. 
Montel, F. (1993). Phase equilibria needs for petroleum exploration and production industry. Fluid Phase Equilibria, 84:343-367.

Montel, F., and P. L. Gouel. (1985). Prediction of Compositional Grading in a Reservoir Fluid Column. Paper presented at the 60th SPE Annual Technical Conference and Exhibition, Las Vegas, NV, September 22-25.

Nikpoor, M. H., R. Kharrat, and Z. Chen. 2011. Modeling of compositional grading in petroleum reservoirs, including the effect of plus fraction properties changes with depth, Petroleum Science and Technology. 29(9):914-923. doi:10.1080/10916460903485884.

Patel, N. C., and A. S. Teja. 1982. A new cubic equation of state for fluids and fluid mixtures. Chemical Engineering Science 37(3):463-473. doi:10.1016/00092509(82)80099-7.

Pederson, S. K., and P. L. Christensen. 2007. Phase behavior of petroleum reservoir fluids. Boca Raton, FL: CRC Press.

Pedersen, K. S., and H. P. Hjermstad. 2006. Modeling of large hydrocarbon compositional gradient. Paper presented at: Society of Petroleum Engineers International Petroleum Exhibition and Conference; November 5-6; Abu Dhabi, U.A.E. doi:10.2118/101275-MS.

Pedersen, K. S., and H. P. Hjermstad. 2015. Modeling of compositional variation with depth for five North Sea reservoirs. Paper presented at: Society of Petroleum Engineers Annual Technical Conference and Exhibition; September 28-30; Houston, Texas. doi:10.2118/175085-MS.

Pedersen, KS, N. Lindeloff. 2003. Simulation of compositional gradients in hydrocarbon reservoirs under the influence of a temperature gradient. Paper presented at: Society of Petroleum Engineers Annual Technical Conference and Exhibition; Oct. 5-8; Denver, Colorado.

Peng, D.-Y., and D. B. Robinson. 1976. A new two-constant equation of state. Industrial and Engineering Chemistry Fundamentals 15(1):59-64. doi:10.1021/i160057a011.

Péneloux, A., E. Rauzy, and R. Fréze. 1982. A consistent correction for RedlichKwong-Soave volumes. Fluid Phase Equilibria 8(1):7-23. doi:10.1016/03783812(82)80002-2.

Privat, R., J.-N. Jaubert, and Y. L. Guennec. 2016. Incorporation of a volume translation in an equation of state for fluid mixtures: which combining rule? which effect on properties of mixing? Fluid Phase Equilibria 427: 414-420. doi:10.1016/j.fluid.2016.07.035.

Redlich, O., and J. N. S. Kwong. 1949. On the thermodynamics of solutions. V. An equation of state. Fugacities of gaseous solutions. Chemical Reviews 44 (1):233-244. doi:10.1021/cr60137a013.

Robinson, D. B., and D.-Y. Peng. 1978. The characterization of the heptanes and heavier fractions for the GPA Peng-Robinson Programs. Tulsa, Oklahoma: GPA Research Report, RR, Vol. 28.

Sage, B. H., and W. N. Lacey. 1939. Gravitational Concentration Gradients in Static Columns of Hydrocarbon Fluids. Transactions of the AIME 132(1):120-131. doi:10.2118/939120-G.

Schmidt, G., and H. Wenzel. 1980. A modified van der waals type equation of state. Chemical Engineering Science 35(7):1503-1512. doi:10.1016/0009-2509(80)800443. 
Schulte, A. M. 1980. Compositional variations within a hydrocarbon column due to gravity. Paper presented at: Society of Petroleum Engineers Annual Technical Conference and Exhibition; September 21-24; Dallas, Texas. doi:10.2118/9235-MS.

Soave, G., 1972. Equilibrium constants from a modified Redlich-Kwong equation of state. Chemical Engineering Science 27(6):1197-1203. doi:10.1016/0009-2509(72) 80096-4.

Span, R., W. Wagner, E. W. Lemmon, and R. T. Jacobsen. 2001. Multiparameter equations of state - recent trends and future challenges. Fluid Phase Equilibria 183184: 1-20. doi:10.1016/S0378-3812(01)00416-2.

Thiele, E. 1963. Equation of the state for hard spheres. The Journal of Chemical Physics 39(2):474-479. doi:10.1063/1.1734272.

Tian, J., and Y. Gui. 2003. Modification to the Van der Waals equation of state. Journal of Phase Equilibria 24(6):533-541. doi:10.1361/105497103772084570.

Tsai, J.-C., and Y.-P. Chen. 1998. Application of a volume-translated Peng-Robinson equation of state on vapor-liquid equilibrium calculations. Fluid Phase Equilibria 145(2):193-215. doi:10.1016/S0378-3812(97)00342-7.

Twu, C. H., D. Bluck, J. R. Cunningham, and J. E. Coon. 1991. A cubic equation of state with a new alpha function and a new mixing rule. Fluid Phase Equilibria 69: 33-50. doi:10.1016/0378-3812(91)90024-2.

Valderrama, J. O. 1990. A generalizes Patel Teja equation of state for polar and nonpolar fluids and their mixtures. Journal of Chemical Engineering of Japan 23(1):87-91. doi:10.1252/jcej.23.87.

Watson, P., M. Casella, D. May, S. Salerno, and D. Tassios. 1986. Prediction of vapor pressures and saturated molar volumes with a simple cubic equation of state: Part II: The Van der Waals - 711 EOS. Fluid Phase Equilibria 27: 35-52. doi:10.1016/03783812(86)87039-X.

Wei, Y. S., and R. J. Sadus. 2000. Equations of state for the calculation of fluid-phase equilibria. AIChE Journal. 46(1):169-196. doi:10.1002/aic.690460119.

Whitson, C. H. and P. Belery. 1994. Compositional gradients in petroleum reservoirs. Paper presented at: University of Tulsa Centennial Petroleum Engineering Symposium; August 29-31; Tulsa, OK. doi:10.2118/28000-MS.

Yu, J.-M., and B. C.-Y. Lu. 1987. A three parameter cubic equation of state for asymmetric mixture density calculations. Fluid Phase Equilibria 34(1):1-19. doi:10.1016/0378-3812(87)85047-1.

Zudkevitch, D., and J. Joffe. (1970). Correlation and prediction of vapor-liquid equilibria with the redlich-kwong equation of state. AIChE Journal, 16(1):112-119. 